\title{
Modeling velocity in gradient flows with coupled-map lattices with advection
}

\author{
Pedro G. Lind, ${ }^{1,2,3, *}$ João Corte-Real, ${ }^{1,3, \dagger}$ and Jason A. C. Gallas ${ }^{1,2,3,4, \$}$ \\ ${ }^{1}$ Unidade de Meteorologia e Climatologia, Instituto de Ciência Aplicada e Tecnologia, Faculdade de Ciências, Universidade de Lisboa, \\ 1749-016 Lisboa, Portugal \\ ${ }^{2}$ Instituto de Física, Universidade Federal do Rio Grande do Sul, 91501-970 Porto Alegre, Brazil \\ ${ }^{3}$ Centro de Geofísica, Universidade de Évora, 7000 Évora, Portugal \\ ${ }^{4}$ Institut für Computer Anwendungen, Universität Stuttgart, Pfaffenwaldring 27, D-70569 Stuttgart, Germany
}

(Received 11 March 2002; published 29 July 2002)

\begin{abstract}
We introduce a simple model to investigate large scale behavior of gradient flows based on a lattice of coupled maps which, in addition to the usual diffusive term, incorporates advection, as an asymmetry in the coupling between nearest neighbors. This diffusive-advective model predicts traveling patterns to have velocities obeying the same scaling as wind velocities in the atmosphere, regarding the advective parameter as a sort of geostrophic wind. In addition, the velocity and wavelength of traveling wave solutions are studied. In general, due to the presence of advection, two regimes are identified: for strong diffusion the velocity varies linearly with advection, while for weak diffusion a power law is found with a characteristic exponent proportional to the diffusion.
\end{abstract}

DOI: 10.1103/PhysRevE.66.016219

PACS number(s): 05.45.Ra, 42.68.Ay, 92.60.Gn, 92.60.Bh

\section{INTRODUCTION}

The aim of the present paper is to introduce a simple model for studying a number of dynamical features observed in atmospheric circulation systems. While still preserving nonlinear effects, the motivation of the model is to simplify the familiar description based on the differential equation underlying the dynamics, Eq. (1) below, in order to spare computation time. For a number of applications, a reduced time of computation opens the possibility for investigating single atmospheric scenarios over much larger time spans, over decades, centuries, or beyond, and allows evaluating average behaviors over larger ensembles of individual scenarios. In addition, fast-running algorithms are very handy in ruling out unrealistic processes.

As is well known [1-3], in setting up models of atmospheric circulation it is common to use sets of spatially continuous orthogonal functions to obtain a discrete and finite set of numbers, a lattice, which approximates in every atmospheric layer the fields corresponding to each of the dependent variables (wind components, temperature, etc.). Horizontal derivatives are then expressed as linear combinations of the horizontal derivatives of the orthogonal functions which, in turn, are approximated by linear combinations of the same functions. In general, atmospheric and oceanographic forecasts based in partial differential equations discretized on such lattices involve quite elaborate computations [1-7]. However, an alternative elegant way to study physical processes is by using a different type of lattice, one where the clock ticks with longer intervals, the so-called coupled-map lattices [8].

Coupled-map lattices (CMLs) were introduced in the early 1980s in investigations of spatiotemporal chaos. Since

\footnotetext{
*Email address: http://www.icat.fc.ul.pt/umc/plind

${ }^{\dagger}$ Email address: jcr@fc.ul.pt

${ }^{\ddagger}$ Email address: http://www.if.ufrgs.br/ jgallas
}

then, they have been successfully applied to a plethora of fields involving spatiotemporal complexity such as fluid dynamics, optics, chemical reactions, plasma, biology, etc. [8]. CMLs have even been used to simulate quantum field theories [9]. By far the most popular CML model is that of a diffusive lattice of quadratic (logistic) maps $[8,10]$. CMLs describe the collective behavior of a (usually quite large) number of interacting low-dimensional local dynamical systems placed on a discrete spatial lattice. So, in addition to the internal dynamics of individual "units," or "cells," CMLs are also characterized by the very rich collective dynamics arising from the coupling between units.

In a seminal work introducing a general procedure for constructing low-order models of atmospheric circulation, Lorenz [2] explains that "The most prominent nonlinear terms in the [equations governing the behavior of the atmosphere], and the only ones appearing in some of the most popular simplifications, represent the advection of some variable quantity .... The terms are therefore quadratic, containing products of the advected quantities with the advecting wind. They cannot be removed by any transformation of the independent or dependent variables." Thus, these arguments clearly show that any realistic attempt at forecasting the evolution of atmospheric systems must incorporate advection $[2,11,12]$.

The basic physical process underlying standard CML models of today is the diffusion among individual cells [8] and, therefore, to use CMLs as models of atmospheric systems one needs first to extend them to include advection. Here we show that a discretization of the appropriate differential equation leads very naturally to a slight generalization of the standard (purely diffusive) CML model, a generalization which is quite appealing as a model of atmospheric systems.

The paper is organized as follows. In Sec. II we start by deriving the model. In Sec. III the velocity of traveling pattern solutions is studied and the numerical results are compared with those obtained for atmospheric fluids such as gra- 
dient flows. Section IV presents a study of the more particular solutions, called traveling waves. Here we emphasize the variation of their wavelengths with advection. A final discussion and conclusions are given in Sec. V.

\section{THE DIFFUSIVE-ADVECTIVE CML MODEL}

The purpose of this section is to introduce the diffusiveadvective CML model. This is done by extending the standard diffusive CML model to incorporate advection. To this end we discretize in space and time the general equation controlling the time evolution of any given intensive physical property $\mathcal{P}$, namely,

$$
\frac{\partial \mathcal{P}}{\partial t}=\mathcal{K} \vec{\nabla}^{2} \mathcal{P}-\vec{v} \cdot \vec{\nabla} \mathcal{P}+\mathcal{F}\left(\mathcal{P}, \mathcal{P}^{\prime}, \ldots\right),
$$

where $\vec{v}$ is the advective velocity and $\mathcal{K}$ the diffusion coefficient [13]. The functional $\mathcal{F}$ depends in general on $\mathcal{P}$ and its spatial derivatives and includes all additional contributions.

Assuming for simplicity a single spatial dimension and unitary increments in space and time, a straightforward discretization of Eq. (1) yields

$$
\begin{aligned}
\mathcal{P}_{n, \tau+1}= & (1-2 \mathcal{K}) \mathcal{P}_{n, \tau}+\frac{2 \mathcal{K}-v}{2} \mathcal{P}_{n+1, \tau}+\frac{2 \mathcal{K}+v}{2} \mathcal{P}_{n-1, \tau} \\
& +F\left(\mathcal{P}_{n \pm i, \tau}\right)
\end{aligned}
$$

where $i=0,1,2, \ldots$ and the integers $\tau$ and $n$ label time and position in the lattice, respectively.

For a generic $F\left(\mathcal{P}_{n \pm i, \tau}\right)$, a plausible first approximation is to consider the system as evolving in a "mean field" which acts effectively so as to modify homogeneously the relative weights of $\mathcal{P}_{n, \tau}, \mathcal{P}_{n+1, \tau}$, and $\mathcal{P}_{n-1, \tau}$ in the first three terms of the second member of Eq. (2). This leads to

$$
\mathcal{P}_{n, \tau+1}=(1-\varepsilon) f\left(\mathcal{P}_{n, \tau}\right)+\frac{\varepsilon-\gamma}{2} f\left(\mathcal{P}_{n+1, \tau}\right)+\frac{\varepsilon+\gamma}{2} f\left(\mathcal{P}_{n-1, \tau}\right),
$$

where $\gamma=v$ represents the advection, $\varepsilon=2 \mathcal{K}$ is the coupling parameter representing diffusion, and $f(x)$ stands for the local nonlinear map representing the mean field, in suitable units. In the remainder we consider the rich dynamics of Eq. (3) when the local cells are ruled by the usual quadratic function $f(x)=1-a x^{2}$, with periodic boundary conditions $x_{L+n}=x_{n}$, where $L$ is the total number of lattice sites.

If, as usual, one interprets the coefficients of the nonlocal terms in Eq. (3), as coupling parameters varying between 0 (no coupling) and 1 (full coupling), then for $0 \leqslant \varepsilon \leqslant 1$ the allowed interval of $\gamma$ is $-\varepsilon \leqslant \gamma \leqslant \varepsilon$.

Equation (3) defines the model incorporating advection through the parameter $\gamma$ which corresponds to an asymmetry in the coupling with nearest neighbors. In the absence of advection $(\gamma=0)$ Eq. (3) reduces to the familiar diffusive model $[8,14-16]$. For $\gamma= \pm \varepsilon$ Eq. (3) reduces to the socalled one-way coupling model [17]. With minor changes of variable, Eq. (3) contains all previous models which in one way or another have asymmetries in the coupling $[18,19]$.

\section{POWER LAW FOR TRAVELING PATTERNS VELOCITY}

One of the most interesting features of the model is the existence of traveling pattern solutions either in the absence $[8,14,16]$ or in the presence $[20]$ of advection. In this section we study the variation of the velocity of such traveling patterns as a function of both advection $\gamma$ and diffusion $\varepsilon$. A main result is that the velocity has a correspondence with the so-called gradient wind velocity [21] over a certain region in the parameter space.

In atmospheric circulating systems, the gradient wind equation is a solution of the equation of motion describing the balance between the centrifugal force, the pressure gradient force, and the Coriolis force. In other words, the gradient wind is a frictionless wind describing the motion of atmospheric flows in closed trajectories [21]. The presence of a centrifugal force yields different solutions for the gradient wind velocity, depending on the signs of the curvature radius and of the pressure gradient. Some of these solutions are "nonphysical" while the others explain the four types of atmospheric pressure system occurring at the mesoscale, namely, the so-called normal or anomalous highs and normal or anomalous lows $[21,22]$. In the atmosphere, the combination of these pressure systems is determinant for its long term behavior; it is rather difficult to predict their evolution for long periods of time, weeks and beyond [23].

In general, the gradient wind velocity depends on the socalled geostrophic wind velocity [see Eq. (6) below], that is, on the horizontal pressure gradient force. In this section we argue that the advection may be interpreted as the geostrophic velocity.

Without advection, Eq. (3) is known to support traveling wave solutions over a wide range of parameters $[8,16]$, namely, $1.6 \leqslant a \leqslant 1.85$ and $0.4 \leqslant \varepsilon \leqslant 0.9$, but always for rather low velocities, of about $v \sim 10^{-3}$ sites/step. Two important effects of the advection here are (i) to induce movement in patterns which would be otherwise stationary, and (ii) to allow velocities to be easily tuned, up to three orders of magnitude. These effects happen over wide parameter ranges.

Figure 1 shows the velocity $v$ of a pattern traveling on a lattice with $L=64$ sites as a function of the advection $\gamma$, computed during $10^{4}$ steps after a transient of $10^{5}$ steps. The dependence is rather different depending on whether the diffusion is strong or weak, with the transition between these regimes happening for $\varepsilon_{t} \sim 0.27$.

As illustrated by Fig. 1(a), for weak diffusion there is a clear domain where the velocity is locked at $v=0$ for an interval $-\gamma_{c} \leqslant \gamma \leqslant \gamma_{c}, \gamma_{c}$ being the maximum value of $\gamma$ for which velocity locking occurs. Outside this locking interval the velocity is well fitted by a power law

$$
v=\frac{\gamma}{|\gamma|}\left(|\gamma|-\gamma_{c}\right)^{\alpha}-\theta
$$

where $\alpha$ depends on the diffusion $\varepsilon$ and the constant $\theta$ represents small fluctuations around the best fit. From the small scale behavior shown in Fig. 1(b) one recognizes that $\theta$ var- 

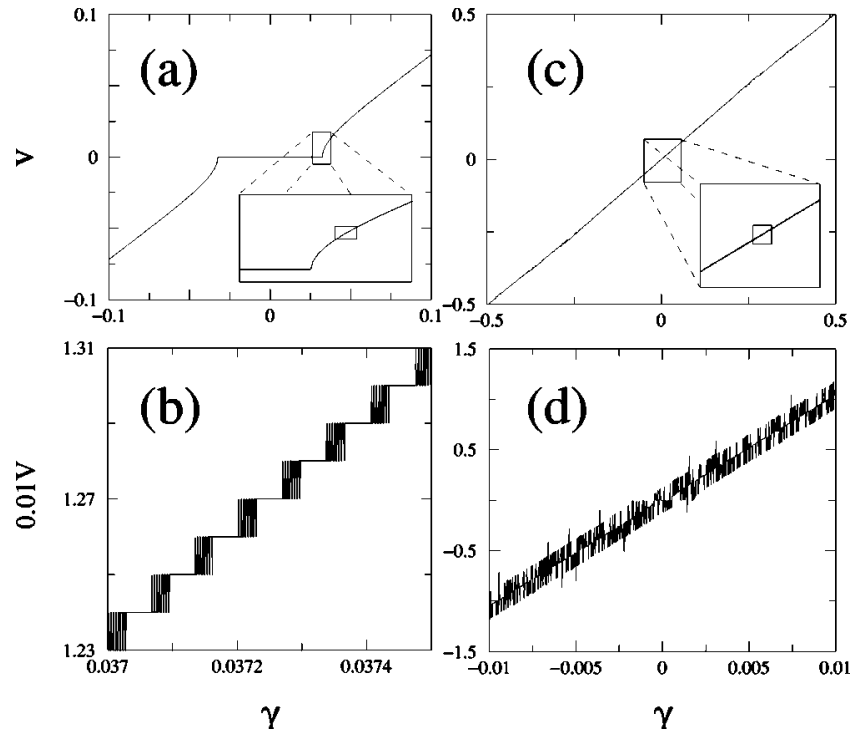

FIG. 1. Velocity dependence on the advection for (a) weak diffusion $(\varepsilon=0.1)$ and periodic local dynamics $(a=1)$; (b) zoom of (a) showing step-function fluctuations; (c) strong diffusion $(\varepsilon$ $=0.5$ ) and chaotic local dynamics $(a=1.7)$; (d) zoom of (c) showing small fluctuations about $v=\gamma$. Here $L=64$.

ies like a step function and that the velocity "hesitates" between different constant values which are roughly equally spaced.

For strong diffusion Eq. (4) no longer applies, the velocity then varying linearly with $\gamma$ [see Fig. 1(c)], apart from a small fluctuation around $v=\gamma$. This fluctuation may be either random, for chaotic local maps [see Fig. 1(d)], or vary in steps, for periodic local maps. Important here is that, despite the strong diffusion, advection dominates diffusion completely with the numerical value of $\gamma$ being a very good first approximation of the velocity value. This is in perfect agreement with the identification of $\varepsilon$ in Eq. (3). In addition, our results show that no power law holds for chaotic local maps, independently of the diffusion strength. Since we are interested in power-law behaviors, we concentrate henceforth only on periodic local maps. For a more detailed study of chaotic coupled maps see Ref. [20].

Figure 2 illustrates the transition from weak to strong diffusion, showing the velocity dependence on both parameters, advection $\gamma$ and diffusion $\varepsilon$, for $a=1$, when the local maps oscillate with period 2. As illustrated in the tridimensional plot in Fig. 2(a), the velocity shows axial symmetry around $\gamma=0$, namely, $v(\gamma, \varepsilon)=-v(-\gamma, \varepsilon)$. Furthermore, one sees the full region of velocity locking at $v=0$. This region is emphasized in Fig. 2(b) which shows the projection of $v$ into the $\varepsilon \times \gamma$ subspace. The maximum value $\gamma_{c}$ of the velocity locking region decreases when diffusion gets stronger. A similar projection into the $v \times \gamma$ subspace is illustrated in Fig. 2(c), suggesting that the exponent $\alpha$ in Eq. (4) increases up to $\sim 1$ for $\varepsilon_{t}$. Beyond this threshold the velocity is not locked anymore but depends linearly on the advection, as shown in Fig. 3.

Figure 3 illustrates not only the power-law behavior of the velocity, but also the variation of the exponent $\alpha$ as a func-
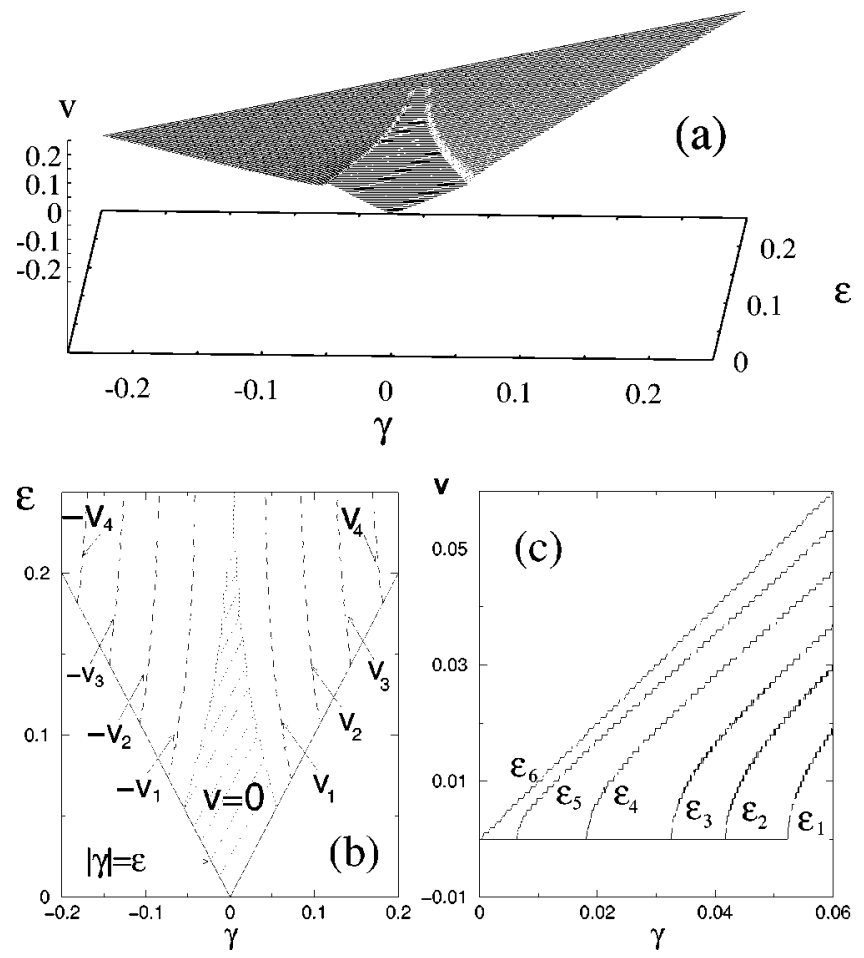

FIG. 2. The transition from weak $(\varepsilon \lesssim 0.27)$ to strong ( $\varepsilon$ $\gtrsim 0.27)$ diffusion, for $a=1$. (a) Symmetry $v(\gamma, \varepsilon)=-v(-\gamma, \varepsilon)$ and locking region $v=0$; (b) projection of $v$ onto the $\gamma \times \varepsilon$ subspace, emphasizing the locking region and showing lines of constant $v, v_{1}=0.04, v_{2}=0.08, v_{3}=0.12$, and $v_{4}=0.16$; (c) the velocity as a function of $\gamma$ for $\varepsilon_{1}=0.06, \varepsilon_{2}=0.08, \varepsilon_{3}=0.1, \varepsilon_{4}=0.14$, $\varepsilon_{5}=0.2$, and $\varepsilon_{6}=0.27 \sim \varepsilon_{t}$ (see text). Here $L=64$. Similar results are obtained for other lattice sizes.

tion of diffusion. Figure 3(a) shows $v \times \gamma$ plots similar to those in Fig. 2(c) but on logarithmic scales for 12 different values of the diffusion $\varepsilon \in[0.06,0.58]$. The best fit for these curves is the linear fit as illustrated in Fig. 3(b), where the slope corresponds to the exponent $\alpha$ in Eq. (4). Furthermore, all the fits intersect roughly at the same point $P$ $\equiv\left(\log \Gamma^{*}, \log v^{*}\right) \sim(-3.16,-3.14)$, where we define $\Gamma^{*}$ $=\left|\gamma^{*}\right|-\gamma_{c}$. With these values of $\Gamma^{*}$ and $v^{*}$ we obtain an expression for the lines for any value of $\alpha$, making it possible to write an approximate expression for $\gamma_{c}$, namely,

$$
\gamma_{c}=|\gamma|-\Gamma^{*}\left(\frac{v}{v^{*}}\right)^{1 / \alpha}
$$

Figure 3(c) clearly shows that (i) for the weak diffusion regime, $\varepsilon \leqslant \varepsilon_{t}$, the exponent $\alpha$ is proportional to the diffusion while (ii) for the strong diffusion regime $\alpha \sim 1$. In other words, $\alpha$ is truly a characteristic exponent, and the above Eq. (5) gives an approximate value of $\gamma_{c}$ for any $(\gamma, \varepsilon, v)$ in the region where the power-law behavior exists (see Fig. 2).

So far, we have considered the dynamic effect of advection in the collective behavior of a coupled lattice. However, a particularly interesting situation arises for $\varepsilon \sim 0.06$, where from Fig. 3(c), one finds $\alpha=0.5$. In other words, in this case the velocity $v$ is proportional to $\sqrt{|\gamma|-\gamma_{c}}$, therefore scaling 

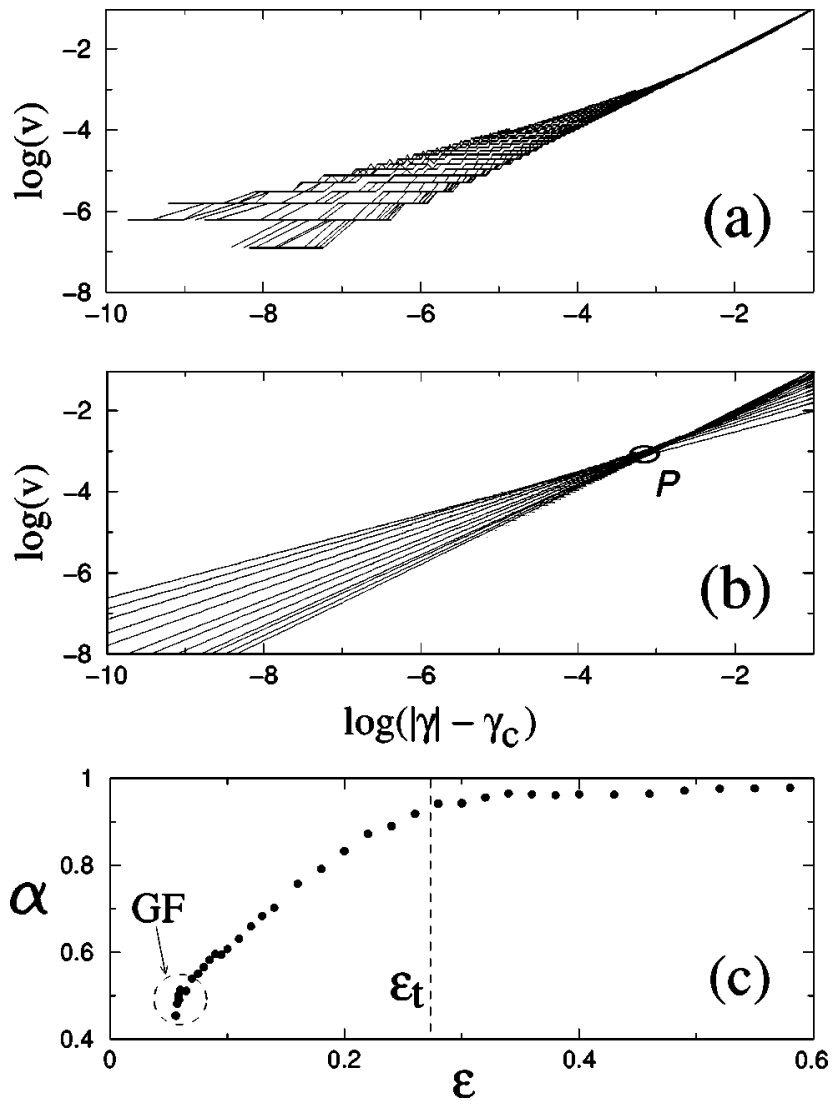

FIG. 3. Variation of the exponent $\alpha$ as a function of the velocity and advection [see Eq. (4)] (a) Log-log plot of the velocity as a function of $\gamma-\gamma_{c}$ and (b) the corresponding fits of the data, where $P \sim(-3.16,-3.14)$ is the approximate intersection of all the lines (see text). (c) Variation of $\alpha$ as a function of $\varepsilon$ showing the transition, at about $\epsilon_{t}=0.27$, from the weak to the strong diffusion regimes. The gradient flow (GF) regime occurs for $\varepsilon \leqq 0.06$ (see text).

precisely as in atmospheric gradient flows, where the wind velocity $V$ displays the same dependence on the geostrophic wind $v_{g}$, namely $[1,3]$,

$$
V= \pm\left(\frac{f^{2} R^{2}}{4}+f R v_{g}\right)^{1 / 2}-\frac{f R}{2}
$$

where $f$ is the Coriolis parameter and $R$ is the radius of curvature. From Figs. 1(a) and 2(c) one also sees that $\gamma_{c} /|\gamma|$ in Eq. (4) is bounded by $0.3 \leqslant \gamma_{c} /|\gamma| \leqslant 1$. Therefore, values in the lower end of this interval have the same order of magnitude as the value $f R /\left(4 v_{g}\right) \sim 0.25$ corresponding to standard mesoscale analysis in middle latitudes where $f \sim 10^{-4} \mathrm{~s}^{-1}$, $R \sim 10^{5} \mathrm{~m}$, and $v_{g} \sim 10 \mathrm{~m} / \mathrm{s}$.

\section{WAVELENGTH MODULATED BY ADVECTION}

In the absence of advection, traveling wave solutions appear to have always the same wavelength $[8,16]$. In this section, we show that in the presence of advection it is possible to change the wavelength of these solutions.

The possibility of predicting traveling wave solutions of different wavelengths in CMLs, implies the existence of dif-

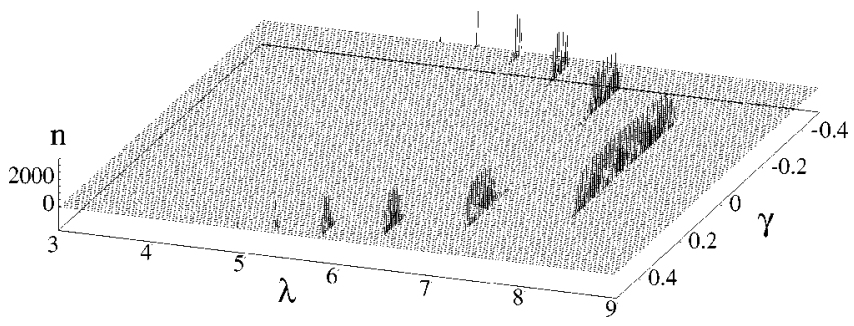

FIG. 4. Wavelength as a function of advection. Histograms were computed from 50 random initial conditions for 100 values of $\gamma$.

ferent spatial scales. In the real atmosphere, atmospheric waves also occur in different spatial scales, generated by local conditions such as topography [24] and influencing the global climate over different spatial scales [25], e.g., the cycle of tides [26] and, as found very recently, the distribution of ozone holes at the earth poles [27]. Most of these atmospheric waves are generated by dynamical processes and interact nonlinearly; they are crucial elements in atmospheric forecasts at all scales.

As recently shown [16], in the absence of advection the region delimited by $1.6 \leqslant a \leqslant 1.85$ and $0.4 \leqslant \varepsilon \leqslant 0.9$ is characterized by the presence of wavelike patterns, i.e., patterns with spatial periodicity $x_{t}(i)=x_{t}(i+k)$ with $k \sim 8$ sites [8]. In this domain, by adding advection, static wavelike patterns start to move while moving patterns increase their velocities; in other words, all patterns turn into traveling waves. We proceed by considering traveling wave solutions of Eq. (3), investigating the spatial dependence of their wavelength on the parameters. Wavelengths were computed from analysis of the spatial correlations among lattice sites [20], namely,

$$
C(i, j)=\frac{\left\langle x_{i} x_{j}\right\rangle-\left\langle x_{i}\right\rangle\left\langle x_{j}\right\rangle}{\left\langle x_{i}^{2}\right\rangle-\left\langle x_{i}\right\rangle^{2}}
$$

where $i$ and $j$ label different sites on the lattice and $\langle X\rangle$ represents the time average of $X$.

Figure 4 shows the spatial wavelength as a function of $\gamma$ for a set of 50 initial conditions. The wavelength reaches a maximum of nearly eight sites for a certain interval about $\gamma=0$ and decreases when advection is further increased, remaining, however, nearly constant inside certain $\gamma$ intervals. Due to an increase in the propagation velocity, the phase difference measured at a given site between successive time steps increases, leading to an increase in the quantity of waves needed to ensure stability of the pattern. This increase in the quantity of waves occurs discontinuously, producing "jumps" in $\lambda$, which correspond to a splitting of the wave number $k$ from $n$ to $n+1$. Thus, the net effect of an increase in the advection is to produce a decrease in the observed wavelengths. The association of stronger advection with smaller wavelengths is also true in the real atmosphere where, for example, the advection of vorticity (a rather important mechanism in cyclogenesis) increases with the square of the wave number [21].

The characteristic values $\gamma_{\ell}$ where the wavelength splits from one value to another depend linearly on $\lambda$, as may be 

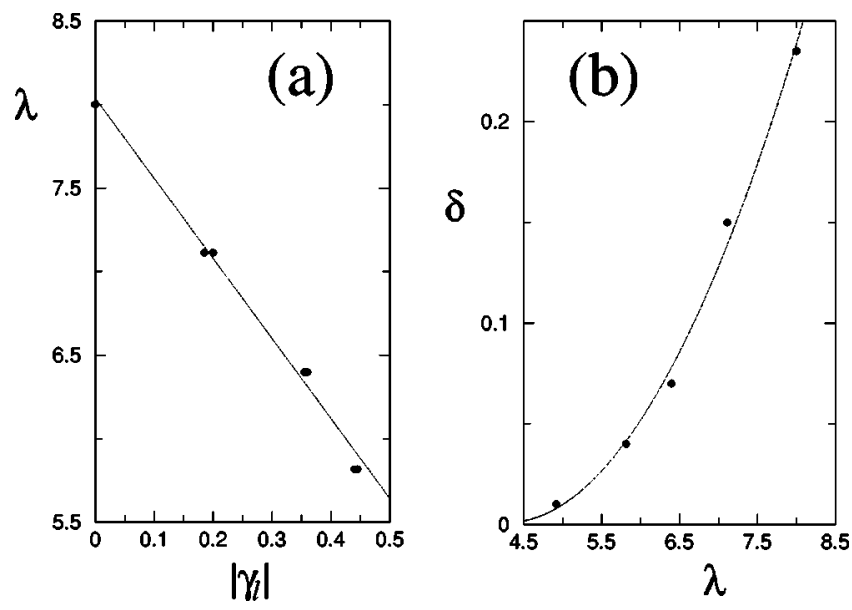

FIG. 5. (a) The critical values $\gamma_{\ell}$, where wavelength transitions occur, vary linearly with $\lambda$; (b) the amplitudes $\delta$ of the $\gamma$ intervals where $\lambda$ is constant are proportional to $\lambda^{2}$.

seen from Fig. 5(a). Figure 5(b) shows that the width $\delta$ of the intervals where the wavelength remains constant is proportional to $\lambda^{2}$.

Both quantities $\gamma_{\ell}$ and $\delta$ depend on diffusion $\varepsilon$. Figure 6 shows a rough illustration of $\gamma_{\ell}$ as a function of both $\gamma$ and $\varepsilon$. The wavelength remains constant inside the parabolicshaped regions and changes abruptly at the boundaries.

\section{CONCLUSIONS}

In conclusion, we have shown that the discretization of the diffusion and advection operators leads directly and quite naturally to a simple generalization of the standard coupledmap lattice model. The model incorporating advection turns out to be particularly well suited for studying dynamical aspects of the atmosphere. For instance, in the weak diffusion regime the velocities of the patterns on the lattice are found to be of the same order of magnitude and to have the same scaling as wind velocities of gradient flows in the atmosphere. In general, there is (i) a weak diffusion regime characterized by a locking region $v=0$ inside an interval centered at $\gamma=0$ and, outside that interval, by an exponent $\alpha$ which describes power-law behavior between the velocity and advection and (ii) a strong diffusion regime where traveling wave solutions have a velocity approximately equal to the value of advection. The latter, also characterized by wavelike solutions, shows characteristic wavelengths in certain ranges of advection values.

All aforementioned results are robust to variations of initial conditions and length of the transients. For instance, the hesitation (the analog of the atmospheric vacillation) between two successive steps seen for weak diffusion in Fig. 1(b) does not depend on the transient used. Furthermore,

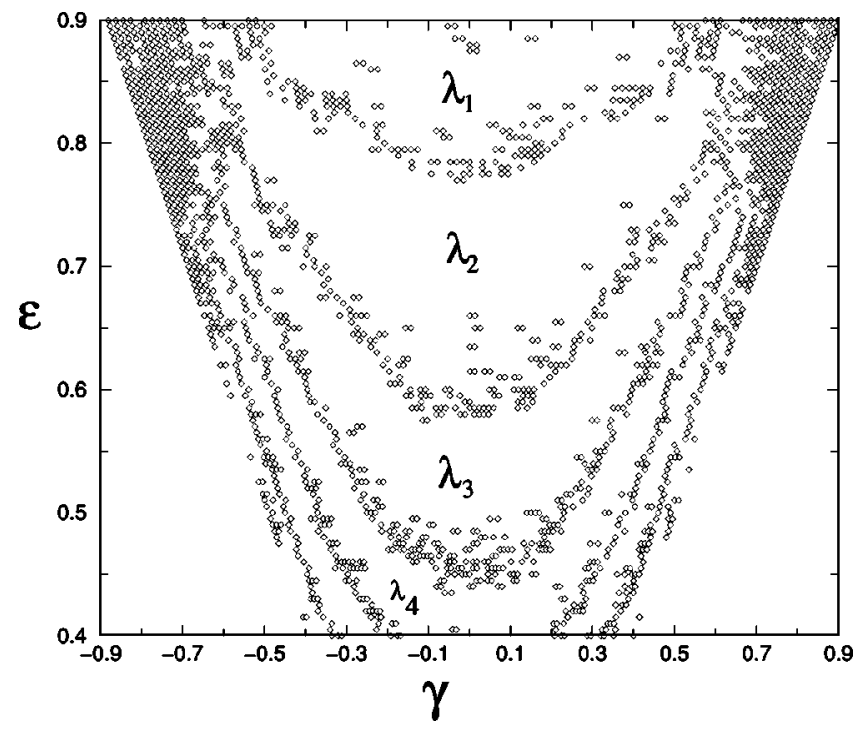

FIG. 6. Wavelength plateaus characterized by constant wavelengths $\lambda_{i}$. At the roughly parabolic separatrices wavelengths change discontinuously: $\lambda_{1}>\lambda_{2}>\lambda_{3}>\cdots$. Here $L=64$ and $a$ $=1.7$.

although in the present work we concentrated on a fixed lattice size, $L=64$, all results are also observed in lattices of different sizes. In fact, the spatial wavelength tends to a finite value in the thermodynamic limit $L \rightarrow \infty$. A more detailed discussion of advection is presented elsewhere [20]. Among others, two questions arise from this study, the first concerning the conditions for velocity locking and the second concerning the possibility to turn periodic patterns that are chaotic for the purely diffusive CML model, by adding advection. These questions are treated elsewhere [28].

As it is not difficult to realize, the present model is now ready to be applied to simulations of two-dimensional lattices having a "vertical" coordinate, in order to verify if traveling wave solutions governed by the advection have velocities and wavelengths lying on a range compatible with the corresponding ones found for atmospheric waves. The inclusion of this additional degree of freedom produces a whole class of models sharing much in common with twolevel models $[1,21,29]$ widely used in atmospheric forecasts, allowing, in particular, the simulation of atmospheric $[28,30]$ and ocean [31] convection.

\section{ACKNOWLEDGMENTS}

This work was supported by Project No. 077/2001 sponsored by CAPES (Brazil) and ICCTI (Portugal). P.G.L. was supported by Fundação para a Ciência e a Tecnologia, Portugal. J.A.C.G. acknowledges support by the Conselho Nacional de Desenvolvimento Científico e Tecnológico, Brazil.
[1] G.J. Haltiner and R.T. Williams, Numerical Prediction and Dynamic Meteorology (John Wiley, New York, 1980).

[2] E.N. Lorenz, J. Meteorol. Soc. Jpn. 60, 255 (1982).
[3] J.P. Peixoto and A.H. Oort, Physics of Climate (American Institute of Physics, New York, 1992).

[4] G. Siedler, J. Church, and J. Gould, Ocean Circulation and 
Climate: Observing and Modeling the Global Ocean (Academic Press, New York, 2001).

[5] The Oceans and Rapid Climate Change: Past, Present, and Future, Geophysical Monograph No. 126, edited by D. Seidov, B.J. Haupt, and M. Maslin (American Geophysical Union, Washington, DC, 2001).

[6] Wind Stress over the Ocean, edited by I. Jones and Y. Toba (Cambridge University Press, Cambridge, England, 2001).

[7] X.B. Zeng, R.A. Pielke, and R. Eykholt, Bull. Am. Meteorol. Soc. 74, 631 (1993).

[8] K. Kaneko and I. Tsuda, Complex Systems: Chaos and Beyond (Springer, Berlin, 2000), and references therein.

[9] C. Beck, Nonlinearity 8, 423 (1995); A. Hilgers and C. Beck, Physica D 156, 1 (2001).

[10] Lattice Dynamics, Proceedings of the Workshop "Lattice Dynamics" (Paris, 1995), edited by H. Chaté and M. Courbage Physica D 103, 1 (1997).

[11] A. Gluhovsky and C. Tong, Phys. Fluids 11, 334 (1999).

[12] A. Gluhovsky and E. Agee, J. Atmos. Sci. 54, 768 (1997).

[13] L.D. Landau and E.M. Lifshitz, Course of Theoretical Physics, Vol. VI-Fluid Mechanics (Pergamon Press, New Delhi, 1959).

[14] P.G. Lind, J. Corte-Real, and J.A.C. Gallas, in Space Time Chaos: Characterization, Control and Synchronization, edited by S. Boccaletti, J. Burguete, W. Gonzalez-Viñas, H. C. Mancini, and D. Valladares (World Scientific, Singapore, 2001), pp. 61-77.

[15] P.G. Lind, J. Corte-Real, and J.A.C. Gallas, Physica A 295, 297 (2001).

[16] P.G. Lind, J. Corte-Real, and J.A.C. Gallas, Int. J. Bifurcation Chaos Appl. Sci. Eng. 11, 2647 (2001).

[17] K. Konishi, H. Kokame, and K. Hirata, Phys. Rev. E 62, 384
(2000); M. Falconi, D. Vergni, and A. Vulpiani, Physica D 125, 65 (1999); F.H. Willeboordse and K. Kaneko, Phys. Rev. Lett. 73, 533 (1994).

[18] Y. Jiang and P. Parmananda, Phys. Rev. E 57, R2499 (1998); Y. Jiang, Phys. Lett. A 240, 60 (1998); K. Kaneko, ibid. 111, 321 (1985).

[19] I. Aranson, D. Golomb, and H. Sompolinsky, Phys. Rev. Lett. 68, 3495 (1992); I.S. Aranson, A.V. Gaponov-Grekhov, and M.I. Rabinovich, Physica D 33, 1 (1988).

[20] P.G. Lind, J. Corte-Real, and J.A.C. Gallas Physica D (to be unpublished).

[21] J.R. Holton, Introduction to Dynamic Meteorology (Academic Press, New York, 1992).

[22] H. Paeth, A. Hense, and R. Glowienka-Hense, Climate Dyn. 15, 953 (1999).

[23] G. Eshel and B.F. Farrel, J. Atmos. Sci. 57, 3219 (2000); J.M. Fritsch and C.F. Chappel, ibid. 37, 1722 (1980); 37, 1734 (1980), and references therein.

[24] J. Röttger, Ann. Geophys. 18, 750 (2000).

[25] L. O'Hanlon, Nature (London) 415, 360 (2002).

[26] A.N. Serebryanya, Dyn. Atmos. Oceans 23, 393 (1996).

[27] P.L. Barry and T. Phillips, http://science.nasa.gov/headlines/ y2001/ast11oct_1.htm

[28] P.G. Lind, J. Corte-Real, and J.A.C. Gallas (unpublished).

[29] V.I. Nekorkin, V.B. Kazantsev, and M.G. Velarde, Physica D 151, 1 (2001).

[30] U. Ulbrich, M.C. Cristoph, J.G. Pinto, and J. Corte-Real, Int. J. Climat. 19, 379 (1999).

[31] P. Lind, S. Titz, T. Kuhlbrodt, J.A.M. Corte-Real, J. Kurths, J.A.C. Gallas, and U. Feudel (unpublished). 